\title{
Analytical reinterpretation of ATLAS dark matter mediator searches with final-state jets
}

\author{
Eric Edward Corrigan ${ }^{* \dagger}$ \\ Lund University \\ E-mail: eric.edward.corrigan@cern.ch
}

Understanding dark matter (DM) is one of the main challenges in modern high-energy physics and cosmology. Alongside direct and indirect detection experiments, one could also produce and discover DM in collider experiments, assuming there is some interaction between DM and Standard Model particles. One form this interaction could take is one mediated by a new vector or axial-vector boson. Furthermore, if DM can be resonantly produced by such a mechanism, the mediator itself could also be discovered by its decay back into quarks. One such search, using the LHC and the ATLAS detector, for jets with an associated photon in the final state, is described, together with an analytical method for the reinterpretation of non-discovery limits, for model parameters different from those used in simulation.

XXIX International Symposium on Lepton Photon Interactions at High Energies - LeptonPhoton2019 August 5-10, 2019

Toronto, Canada

* Speaker.

${ }^{\dagger}$ on behalf of the ATLAS Collaboration 


\section{ATLAS low-mass dark matter mediator searches}

Dark matter (DM) searches at collider experiments commonly take the form of searching for undetectable DM particles as missing transverse momentum $p_{\mathrm{T}}$ in an event. However, in models where DM interacts with Standard Model (SM) particles via exchange of a new gauge boson [4], resonant production of this mediator, and its subsequent decay back to quarks, can be a powerful probe of a new interaction connecting the SM and DM. At the LHC, the ATLAS detector [1] is used to conduct a broad search programme for this class of DM mediator model. At low mediator masses, well below $1 \mathrm{TeV}$, the large QCD multijet background is particularly challenging for searches that target hadronic final states. In particular, unprescaled triggers have much too high $p_{\mathrm{T}}$ thresholds to probe mediators with masses much lower than $1 \mathrm{TeV}$. Several strategies exist to circumvent this limitation; here we will focus on a search which requires an associated high- $p_{\mathrm{T}}$ object (photon) in the final state [7]. This effectively lowers the event rate, reducing the signal rates but allowing access to very low- $p_{\mathrm{T}}$ jets while still using unprescaled triggers. This simplifies the analysis and increases its sensitivity.

\section{The resolved dijet+ISR search}

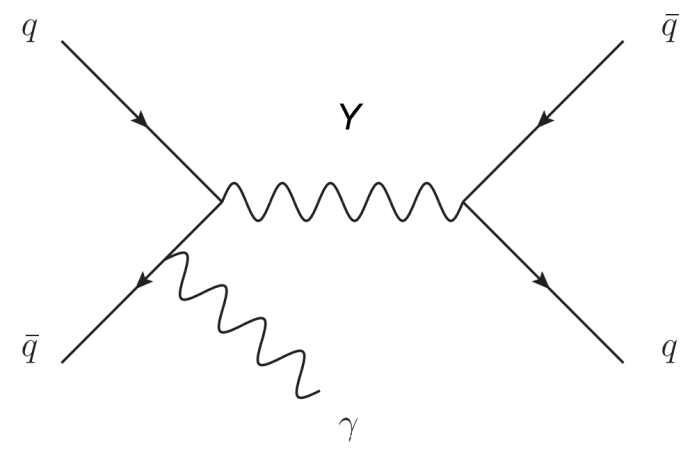

Figure 1: The event topology considered by the resolved dijet+ISR analysis [7], for a new vector or axialvector boson mediator $Y$.

The search [7] considers the event topology shown in Figure 1, with a resonance decay into quarks and associated photon initial-state radiation (ISR). When the mediator mass is very small, the di-quark system becomes boosted, and the merged jets can be treated as a single large-radius jet. Here we will present the resolved analysis, where the two jets are individually resolvable by the detector. In addition to the flavour-inclusive case, where no requirements are placed on the flavour of the quarks $q$, the di- $b$-quark case was also studied, which has comparable sensitivity to the flavour-inclusive (with universal quark-mediator couplings). For mediator masses below $450 \mathrm{GeV}$, a single-photon trigger was used, whereas a multi-object (compound) trigger, requiring one photon and two jets, was used above $450 \mathrm{GeV}$. These data were analysed by fitting the dijet invariant mass spectrum with the best of several considered functional forms, looking for excesses above the fit. No signal was observed, and exclusion limits like those in Figure 2 were computed, quantifying the regions of $95 \%$ CL exclusion of generic Gaussian signal shapes and the benchmark $Z^{\prime}$ model in [4]. 

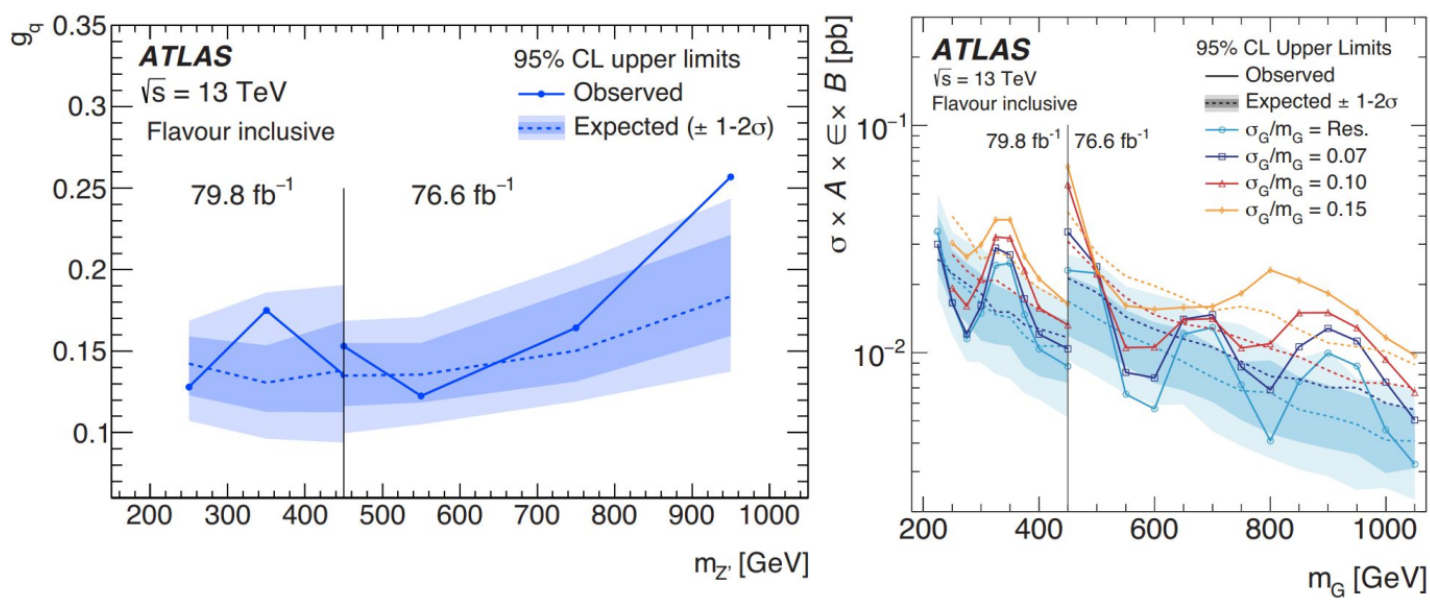

Figure 2: Exclusion limits on the benchmark $Z^{\prime}$ model (left) and generic Gaussian signals (right) for the flavour-inclusive channel [7]. The Gaussian limits are presented for four choices of relative widths, where "Res." is detector dijet mass resolution (8-3\% across the mass range presented).

\section{Limit reinterpretation}

In order to summarize findings such as the above, and to visualize the level of complementarity of the search programme, analysis exclusion limits are compiled in summary plots (like Figure 3). However, analyses' specific choices of models and parameters used to express the exclusion limits are in general not the same. Thus, in order to express the various results in the same plot and model space, the results need to be reinterpreted in a common plane of parameters with the same assumptions and values of the other variables. Traditionally, for ATLAS DM mediator dijet searches, this has been done by producing new signal Monte Carlo samples and performing a comparison to the Gaussian limits [5]. In addition to shortcomings such as being too conservative in some cases, generating signal samples can take several days, and the reinterpretation process requires significant effort. A purely analytical method, new to ATLAS but used by CMS [6], is presented here. This fixes some shortcomings of the previous method (e.g. being too conservative in certain situations), running in less than a second. The central idea is to equate the total excluded cross-sections in the two sets of parameter and assumption choices; that the total cross-section excluded in one set of choices is equal to the total cross-section excluded in the other. This assumes equal acceptance for a given mediator mass in either model, and also that the resonances are narrow; both have been shown to be reasonable assumptions. Let the analysis limits be expressed in the plane of quarkmediator coupling and mediator mass, $\left(g_{q}, M_{\text {med }}\right)$, and be valid for a set of parameters $P$ (such as DM masses $m_{\mathrm{DM}}$, DM and lepton couplings $g_{\mathrm{DM}}, g_{l}$, etc). The summary plot limits should be in the $\left(m_{\mathrm{DM}}, M_{\mathrm{med}}\right)$ plane and evaluated at a set of parameters $Q$. The central equation is then

$$
\sigma_{\text {analysis }}^{\text {excluded }}\left(g_{q}, M_{\text {med }}, P\right)=\sigma_{\text {reinterpreted }}^{\text {excluded }}\left(m_{\mathrm{DM}}, M_{\text {med }}, Q\right)
$$

The cross-sections can be expressed in terms of the partial and total mediator widths. For relativistic Breit-Wigner resonances, this relationship is, in the approximation of narrow widths,

$$
\sigma \approx \frac{\Gamma_{\text {initial }} \Gamma_{\text {final }}}{\Gamma_{\text {total }}}
$$


where $\Gamma_{\text {initial(final) }}$ are the partial widths in the initial (final) states (e.g. $\Gamma_{\text {initial }}=\Gamma_{\text {final }}=\Gamma_{\mathrm{q} \overline{\mathrm{q}}}$ for the $q \bar{q} \rightarrow Z^{\prime} \rightarrow q \bar{q}$ case). The partial widths are known functions (given in Sect 2.3 in [3]) on the couplings to dark matter, quarks and leptons, and their masses. Using these, both sides of the cross section equation can be evaluated, and the expressions for the cross-sections can be inverted, allowing the equation to be written

$$
g_{q}=f\left(M_{\mathrm{med}}, m_{\mathrm{DM}}, P, Q\right)
$$

where $f$ is some function (containing partial widths). Now, since $P$ and $Q$ are explicitly known (typically particle masses and couplings), one can scan the $\left(m_{\mathrm{DM}}, M_{\mathrm{med}}\right)$ plane, evaluating $f$ at each point, and obtain a $g_{q}$ value for each point. If this is larger than the analysis limits, the point is excluded. Thus, reinterpreted limits have been calculated using only simple analytical relationships, without the Monte Carlo generation and general complexity of old method, in much shorter time.

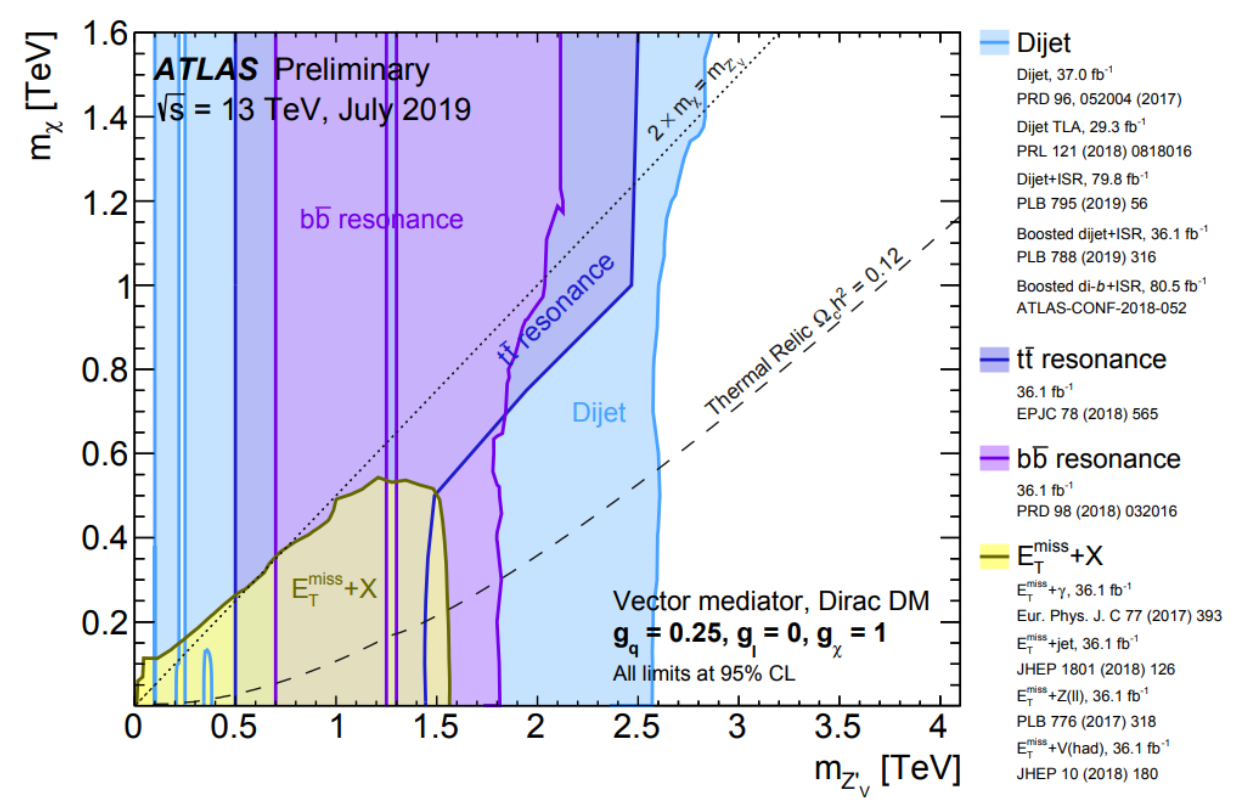

Figure 3: Landscape of ATLAS DM mediator limits expressed in the plane of the (axial-vector) mediator mass and its universal coupling to SM quarks [2].

\section{References}

[1] ATLAS Collaboration, 2008 JINST 3 S08003.

[2] ATLAS Collaboration, ATL-PHYS-PUB-2019-030, https://cds.cern.ch/record/2684864.

[3] A Albert et al. arXiv:1703.05703.

[4] D. Abercrombie et al, arXiv:1507.00966.

[5] ATLAS Collaboration, Phys. Rev. D 91 (2015) 052007.

[6] CMS Collaboration, Phys. Lett. B 778 (2018) 263.

[7] ATLAS Collaboration, Phys. Lett. B 795 (2019) 56. 